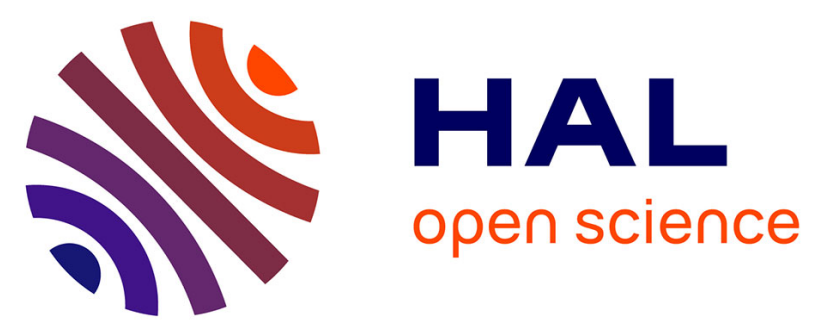

\title{
Inhibiteurs du check-point immunitaire en néo-adjuvant dans les cancers bronchiques non à petites cellules localisés : Essai clinique de phase II IFCT-1601 IoNESCO
}

X. Mignard, M. Antoine, D. Moro-Sibilot, C. Dayen, B. Mennecier, R. Gervais, E. Amour, B. Milleron, F. Morin, G. Zalcman, et al.

\section{To cite this version:}

X. Mignard, M. Antoine, D. Moro-Sibilot, C. Dayen, B. Mennecier, et al.. Inhibiteurs du checkpoint immunitaire en néo-adjuvant dans les cancers bronchiques non à petites cellules localisés : Essai clinique de phase II IFCT-1601 IoNESCO. Revue des Maladies Respiratoires, 2018, 35 (9), pp.983-988. 10.1016/j.rmr.2018.08.006 . hal-01974491

\section{HAL Id: hal-01974491 \\ https://hal.sorbonne-universite.fr/hal-01974491}

Submitted on 8 Jan 2019

HAL is a multi-disciplinary open access archive for the deposit and dissemination of scientific research documents, whether they are published or not. The documents may come from teaching and research institutions in France or abroad, or from public or private research centers.
L'archive ouverte pluridisciplinaire HAL, est destinée au dépôt et à la diffusion de documents scientifiques de niveau recherche, publiés ou non, émanant des établissements d'enseignement et de recherche français ou étrangers, des laboratoires publics ou privés. 
Inhibiteurs du checkpoint immunitaire en néo-adjuvant dans les CBNPC localisés

Sous-titre : Essai clinique de phase II IFCT-1601 IoNESCO
X. Mignard ${ }^{1}$, M. Antoine ${ }^{1,2}$,
D. Moro-Sibilot ${ }^{3,4}$
C. Dayen ${ }^{3,5}$
B. Mennecier ${ }^{3,6}$, R. Gervais
${ }^{3,7}$, E. Amour ${ }^{3}$,
B. Milleron ${ }^{3}$, F. Morin ${ }^{3}$,
G. Zalcman ${ }^{3,8}$, M. Wislez ${ }^{\star 1,3,9}$

1 - Sorbonne Universités, UPMC Univ Paris 06, GRC n04, Theranoscan, F-75252, Paris, France

2 - AP-HP, Hôpital Tenon, Service d'Anatomopathologie et Cytologie, F-75970, Paris, France

3 - IFCT (Intergroupe Francophone de Cancérologie Thoracique), 10 rue de la GrangeBatelière, 75009 Paris, France

4 - CHU de Grenoble, Unité d'Oncologie Thoracique-Pneumologie, Grenoble, France

5 - $\mathrm{CH}$ de Saint-Quentin, Service de Pneumologie, Maladies Infectieuses et Tropicales, BP 608, 02321 Saint-Quentin Cedex, France

6 - CHU de Strasbourg, Service de Pneumologie, F-67000, Strasbourg, France

7 - Centre François Baclesse, Caen, France

8 - AP-HP, Hôpital Bichat-Claude Bernard, Service d'Oncologie Thoracique, Paris, France

9 - AP-HP, Hôpital Tenon, Service de Pneumologie, F-75970, Paris, France

Titre court :

Essai clinique de phase II IFCT-1601 IoNESCO

Auteur correspondant :

Pr Marie Wislez, Service de Pneumologie, Hôpital Tenon, 4 rue de la Chine, 75970 Paris Cedex 20. Email : marie.wislez@aphp.fr. Téléphone : 0156016515. 
rmr170317-R1

Inhibiteurs du checkpoint immunitaire en néo-adjuvant dans les CBNPC localisés

Résumé français (204 mots)

Etat des connaissances: Programmed death-Ligand 1 (PD-L1) est une protéine membranaire facilitant l'échappement tumoral vis-à-vis de l'immunité. Le durvalumab est un anticorps monoclonal bloquant l'interaction PD-L1/PD-1 entre lymphocytes $T$ et cellules tumorales, ayant montré des réponses durables et une toxicité acceptable en phase I/II dans les cancers bronchiques non à petites cellules (CBNPC) métastatiques.

Méthodes: Dans cette étude de phase II, 3 perfusions de durvalumab $(10 \mathrm{mg} / \mathrm{kg}$ à $\mathrm{J} 1$, J15, J29) sont administrées en néoadjuvant à des patients $\geq 18$ ans, indice de performance (PS) 0-1, ayant un CBNPC de stade clinique IB $(>4 \mathrm{~cm})$ ou II). La chimiothérapie et la radiothérapie pré-opératoire ne sont pas permises. L'objectif principal est la faisabilité d'une résection chirurgicale complète. Un taux de résection complète $<85 \%$ (P0) est considéré comme inacceptable. Avec une hypothèse P1 de 95\%, une puissance de $90 \%$ et un risque alpha de 5\%, 81 patients sont nécessaires. La réponse histologique majeure ( $\leq 10 \%$ de cellules tumorales résiduelles sur la pièce opératoire) est un objectif secondaire. Les autres objectifs sont le délai entre traitement et chirurgie, les taux de réponse radiologique et métabolique, les évènements indésirables, la survie sans récidive et globale.

Résultats attendus : L'immunothérapie néo-adjuvante est faisable et pourrait améliorer la survie des CBNPC de stade précoce. 
rmr170317-R1

\section{Mots-clés}

CBNPC, stade précoce, durvalumab, anti-PD-L1, néo-adjuvant

IONESCO : Immune neoajuvant therapy in early stage non-small cell lung cancer

\section{Abstract (230 words)}

Background: Programmed cell death-Ligand 1 (PD-L1) is a checkpoint receptor that facilitates immune evasion by tumor cells, through interaction with PD-1 (Programmed cell death-1), a receptor expressed by T-cells. Durvalumab is an anti-PD-L1 monoclonal antibody that blocks PD-L1 interaction with PD-1 on T-cells, countering the tumor's immune-evading tactics. Phase $\mathrm{I} / \mathrm{II}$ studies demonstrated durable responses and manageable tolerability in heavily pre-treated patients.

Methods: This phase II study is designed to administrate three durvalumab IV infusions (10 $\mathrm{mg} / \mathrm{kg}$ at Day $1,15,29)$ before surgery, to patients with pathologically confirmed nonsmall cell carcinoma (NSCLC), clinical stage IB $(>4 \mathrm{~cm})$ or stage II, $\geq 18$ years of age, WHO performance status $0-1$, without selection on PD-L1 expression. Pre-operative chemotherapy and radiation therapy are not permitted. The primary objective is feasibility of complete surgical resection. Major pathological response on surgical tissue, defined as $10 \%$ or less remaining tumor cells, will be a secondary objective. Additional secondary objectives include tolerance, adverse effects, delay between start of treatment and surgery, response rate (RECIST 1.1), metabolic response rate, post-operative adverse events, disease-free survival and overall survival. A rate of complete resection $<85 \%$ (P0) is considered unacceptable. P1 hypothesis is of $95 \%$, and with a study power of $90 \%$ and an alpha risk of 5\% (two-steps Fleming's procedure), 81 patients are required.

Expected results: To establish whether neo-adjuvant immunotherapy is feasible and could improve the survival of patients with early-stage NSCLC. 
rmr170317-R1

\section{$\underline{\text { Keywords }}$}

NSCLC, early stage, durvalumab, anti-PD-L1, neoadjuvant 
rmr170317-R1

\section{Etat des connaissances}

Le cancer du poumon est la première cause de décès par cancer dans le monde, avec une survie globale (SG) à 5 ans de $17 \%$ environ, tous stades confondus [1]. Le traitement des cancers broncho-pulmonaires non à petites cellules (CBNPC) de stade précoce (I - II) repose sur la chirurgie curative. Bien que les patients traités par résection chirurgicale aient un meilleur pronostic, la survie à 5 ans reste basse, allant de $92 \%$ dans les stades IA1 à 53\% dans les stade IIB [2]. La chimiothérapie adjuvante procure une amélioration de $5 \%$ à 5 ans de la SG chez les patients ayant un CBNPC stade II ou IIIA avec résection complète [3]. La chimiothérapie néo-adjuvante a montré un bénéfice similaire dans plusieurs méta-analyses, dont une sur données individuelles [4]. L'approche néoadjuvante présente plusieurs avantages théoriques : réduction du volume tumoral facilitant le geste opératoire, contrôle rapide de la maladie micro-métastatique, évaluation de la chimio-sensibilité, meilleure compliance que la chimiothérapie adjuvante (qui ne peut être administrée dans plus de la moitié des cas). Un des risques théoriques est la survenue d'une progression tumorale causant un retard du traitement chirurgical, ou le rendant plus compliqué voire impossible.

La réponse tumorale histologique majeure, définie par la présence de $\leq 10 \%$ de cellules tumorales viables après traitement néo-adjuvant par une chimiothérapie à base de sels de platine, est corrélée à la survie $[5,6]$. Ce critère a été proposé dans les essais cliniques comme objectif principal de substitution afin d'obtenir des résultats rapidement et d'éviter plusieurs années de suivi pour l'obtention des évènements [7].

Les points de contrôle immunitaire sont des récepteurs membranaires impliqués dans la régulation de l'activité des cellules immunitaires, notamment des lymphocytes $T$. Physiologiquement, ils régulent négativement cette activité, jugulant le développement de l'auto-immunité. Ils comprennent notamment deux récepteurs distincts : CTLA-4 (Cytotoxic 
rmr170317-R1

T-lymphocyte associated protein 4) et PD-1 (Programmed cell death 1), qui sont exprimés par les lymphocytes $T$, les cellules présentatrices d'antigènes, et des cellules des tissus non lymphoïdes [8]. PD-1 interagit avec PD-L1 (Programmed death-ligand 1), qui peut être exprimé par les cellules tumorales. Une expression membranaire de PD-L1 par les cellules tumorales induit une anergie des lymphocytes $\mathrm{T}$, permettant l'échappement tumoral vis-à vis de la réponse immunitaire [9].

Les inhibiteurs des points de contrôle immunitaire sont des anticorps monoclonaux inhibant l'interaction des points de contrôle avec leur ligand (CTLA-4/B7 et PD-1/PD-L1). Ils comprennent des anticorps anti-CTLA-4 (ipilimumab, tremelimumab), anti-PD-1 (nivolumab, pembrolizumab) et anti-PD-L1 (atezolizumab, avelumab, durvalumab). Ils ont démontré un bénéfice de survie chez les patients atteints de CBNPC métastatique en deuxième puis en première ligne $[10,11,12,13]$. Plus récemment, certains ont montré une efficacité dans les stades localement avancés après chimio-radiothérapie [14].

Leur intérêt dans les stratégies de traitements péri-opératoires doit être évalué, ce qui fait l'objet de cet essai. Ils font par ailleurs l'objet d'essais thérapeutiques en adjuvant dans plusieurs autres néoplasies: mélanome cutané réséqué (nivolumab, [15]), carcinome urothélial réséqué envahissant la musculeuse (atezolizumab, [16]), carcinomes épidermoïdes de la tête et du cou (nivolumab, pembrolizumab, [17]).

Le durvalumab (MEDI4736, Imfinzi®, Astra Zeneca) est un anticorps monoclonal de type IgG1 kappa, anti-PD-L1. L'inhibition de PD-L1 survient à des concentrations d'ordre picomolaire, avec une cytotoxicité hors cible (autres cellules exprimant PD-L1) limitée. Le durvalumab permet l'activation des lymphocytes $T$ in vitro, et induit une réponse tumorale in vivo (xénogreffes tumorales sur des modèles murins syngéniques) [18]. Son efficacité et sa tolérance sont basées sur une étude de phase I (NCT01693562) chez des patients ayant un cancer solide avancé. Des effets secondaires de grade 3 ou 4 ont été rapportés 
rmr170317-R1

chez 10\% des patients (notamment asthénie et hyponatrémie). Les effets secondaires auto-immuns de grade 3 ou 4 (entérocolite, dermite, hépatite, pneumopathie, endocrinopathie) ont été rapportés chez $4 \%$ des patients [19]. Aucune réaction allergique n'a été documentée. La dose recommandée en phase II est de $10 \mathrm{mg} / \mathrm{kg}$ toutes les 2 semaines. Le durvalumab est en cours d'évaluation dans 41 essais cliniques dans les CBNPC, en monothérapie ou en association (https://www.clinicaltrials.gov/ct2/results?cond=NSCLC\&term=MEDI4736). Une étude de phase III (ARCTIC, NCT02352948) est en cours pour évaluer le durvalumab seul ou avec tremelimumab en $3^{\text {ème }}$ ligne chez des patients ayant un CBNPC métastatique [20]. Récemment, dans l'essai de phase III PACIFIC, l'intérêt du durvalumab a été évalué chez 713 patients ayant un CBNPC localement avancé inopérable. Le durvalumab était administré après chimio-radiothérapie concomitante. L'étude est positive avec un bénéfice de survie sans progression (SSP) de 16,8 mois contre 5,6 mois (HR 0,52; IC 95\% [0,420,65], $p<0,001$ ), indépendamment de l'expression de PD-L1 [14]. L'intérêt du durvalumab en adjuvant dans les CBNPC réséqués de stade IB $(\geq 4 \mathrm{~cm})$, II ou IIIA fait l'objet d'une étude de phase III internationale dans laquelle la France est le premier centre en termes d'inclusion (IFCT-1401 BR31, NCT02273375). De même, l'essai clinique PEARLS évalue en phase III le pembrolizumab en adjuvant dans les CBNPC de stade IB à IIIA réséqués (NCT02504372).

Notre essai évalue la faisabilité du Durvalumab en périopératoire et plus précisément en situation néo-adjuvante. 
rmr170317-R1

\section{Méthodes}

II s'agit d'un essai de phase II multicentrique, prospectif, ouvert, mono-bras, évaluant la faisabilité de l'administration de trois injections de durvalumab (MEDI4736), anticorps monoclonal anti-PD-L1, en situation néo-adjuvante au cours des CBNPC résécables de stade IB $(\geq 4 \mathrm{~cm})$ ou II.

\section{$\underline{\text { Population étudiée - critères d'inclusion }}$}

- Patients d'âge supérieur à 18 ans

- Consentement éclairé et écrit recueilli

- Patiente ne pouvant plus procréer ou patiente acceptant de poursuivre une contraception hautement efficace avant la première dose de traitement et jusqu'à 4 mois après la dernière dose de traitement

- Performance status (PS) 0 ou 1

- Paramètres fonctionnels ventilatoires adéquats pour la résection chirurgicale planifiée (lobectomie ou pneumonectomie)

- Diagnostic de CBNPC confirmé histologiquement, de stade IB $(\geq 4 \mathrm{~cm})$ ou II d'après la classification TNM 2015 (8 $8^{\text {ème }}$ édition de l'IASLC). Avant inclusion et chirurgie seront réalisés un scanner thoraco-abdomino-pelvien, un scanner ou une IRM cérébrale, et un TEP-TDM au 18-FDG. Si le scanner et/ou le TEP-TDM montrent une suspicion d'envahissement ganglionnaire, une exploration médiastinale par médiastinoscopie ou cytoponction échoguidée devra être réalisée

- Echantillon de tissu pré-thérapeutique disponible : une coupe HES et un bloc fixé au formol et inclus en paraffine 
rmr170317-R1

- Echantillon de tissu post-thérapeutique prélevé par voie chirurgicale, congelé ou fixé au formol et inclus en paraffine. Au moins une coupe doit être analysée par cm de tumeur dans le plus long diamètre, soit 5 à 30 coupes évaluées. Cela est valable pour la tumeur primitive réséquée mais également pour les adénopathies afin de pouvoir évaluer la réponse histologique

- Echantillons pré et post-thérapeutiques de sérum et de plasma congelé

- Hématologie (dans les 14 jours précédant l'inclusion): hémoglobine $\geq 9,0 \mathrm{~g} / \mathrm{dL}$, polynucléaires neutrophiles $\geq 1500 / \mathrm{mm}^{3}$, plaquettes $\geq 100000 / \mathrm{mm}^{3}$

- Biochimie (dans les 14 jours précédant l'inclusion) : bilirubine totale < LSN (limite supérieure de la normale) sauf syndrome de Gilbert, phosphatases alcalines $\leq 2,5$ LSN, ASAT et ALAT $\leq 2,5 \mathrm{LSN}$, clairance de la créatinine calculée par la formule de Cockroft $\geq 40 \mathrm{ml} / \mathrm{min}, \mathrm{TSH}<\mathrm{LSN}$

\section{$\underline{\text { Population étudiée - critères de non-inclusion }}$}

- Grossesse ou allaitement

- Antécédent de cancer, sauf : cancer traité et guéri depuis plus de 5 ans, carcinome baso-cellulaire cutané, carcinome in situ du col utérin

- Antécédent de traitement par durvalumab ou tout autre anti-PD-1 / anti-PD-L1

- Maladie cardiovasculaire non traitée et/ou non contrôlée et/ou dysfonction cardiaque symptomatique. Les patients présentant des antécédents cardiaques significatifs, même contrôlés, doivent avoir une fraction d'éjection du ventricule gauche $>50 \%$ dans les 12 semaines précédant l'inclusion

- QTc moyen > 470 ms ou antécédent familial de syndrome du QT long

- Pneumopathie interstitielle 
rmr170317-R1

- Neuropathie sévère

- Antécédent de maladie auto-immune, active ou non

- Antécédent de maladie inflammatoire chronique de l'intestin, active ou non

- Antécédent d'immunodéficience innée, de transplantation allogénique d'organe, traitement immunosuppresseur

- Traitement par corticostéroïdes oraux (>10 mg/j d'équivalent-prednisone) moins de 28 jours avant l'inclusion. Les corticostéroïdes inhalés ou nasaux ne constituent pas une contre-indication

- Infection par le VIH

- Infection non contrôlée par le virus de l'hépatite B ou C

- Antécédent de tuberculose active

- Vaccin vivant atténué administré dans les 30 jours précédant la randomisation

- Antécédent d'hypersensibilité au durvalumab ou à l'un de ses excipients

\section{$\underline{\text { Traitement }}$}

- 3 perfusions de durvalumab (MEDI4736), 10 mg/kg, à J1, J15 et J29. Début du traitement dans les 7 jours après l'inclusion

- Résection chirurgicale réalisée entre 2 jours et 2 semaines après la fin du traitement par durvalumab (Figure 1)

- Une chimiothérapie adjuvante par doublet à base de platine pourra être administrée selon le choix du médecin référent

- La chimiothérapie et/ou la radiothérapie pré-opératoire ne sont pas autorisées 
rmr170317-R1

\section{Critère de jugement principal}

- Faisabilité de la résection chirurgicale complète (R0) après immunothérapie. La résection complète est définie par l'absence de résidu tumoral macroscopique ou microscopique sur les marges de résection, avec négativité du premier relais ganglionnaire médiastinal.

\section{Critères de jugement secondaires}

- Toxicité, évènements indésirables (CTCAE v 4.0) du durvalumab

- Délai entre début du traitement et chirurgie

- Taux de réponse radiologique (selon RECIST 1.1 et Immune Response Criteria) après 3 cycles de durvalumab

- Taux de réponse métabolique (TEP-TDM au 18-FDG) après 3 cycles

- Réponse histologique évaluée sur la pièce opératoire, tumeur primitive et ganglions.

- Evènements indésirables post-opératoires

- Survie sans récidive (SSR)

- Survie globale (SG)

- Evaluation de la valeur prédictive/pronostique de l'expression de PD-L1, détectée par immunohistochimie (SP263, Ventana $\left.{ }^{\circledR}\right)$

- Evaluation des variations de cytokines et autres biomarqueurs sériques avant et après traitement 


\section{Analyses statistiques}

Les hypothèses sont:

- I'hypothèse nulle, correspondant à un taux de faisabilité de la chirurgie $\leq 85 \%$, considéré comme inacceptable ( $\mathrm{P} 0=85 \%)$

- I'hypothèse non nulle, $\mathrm{P} 1=95 \%$

Un test de proportion binomial (comparé à une proportion théorique) avec une règle d'arrêt selon O'Brien et Fleming en deux étapes (arrêt de futilité) sera utilisé, avec une puissance de $90 \%$ et un risque alpha de $5 \%$. Le nombre de patients nécessaire est de 81 .

\section{$\underline{\text { Suivi }}$}

Les patients seront suivis par examen clinique et radiographie thoracique tous les 3 mois, et scanner thoracique tous les 6 mois pendant les 2 premières années. Après 2 ans, le suivi comprendra examen clinique et radiographie thoracique tous les 6 mois, et scanner thoracique tous les ans jusqu'à 5 ans au total.

\section{Durée de l'étude}

La période d'inclusion sera de 18 mois et la période de suivi de 1 an. Les inclusions ont débuté le 12 janvier 2017. En date du 16 août 2018, 24 centres ont été ouverts et 37 patients ont été inclus.

\section{Avis du comité d'éthique}

Un avis favorable vis-à-vis du présent essai clinique a été rendu par le Comité de Protection des Personnes d'lle de France (numéro EudraCT : 2016-001849-15, référence CPP : protocole 24-2016). 


\section{Résultats attendus et perspectives}

Un traitement néo-adjuvant par immunothérapie doit démontrer la faisabilité en termes de résection chirurgicale (résection complète, R0), ce qui est l'objectif principal. L'objectif secondaire est le taux de réponses histologiques majeures, qui pourrait être corrélé à une amélioration de la SSR et de la SG.

Des résultats encourageants concernant la faisabilité de l'immunothérapie en schéma néo-adjuvant ont été présentés à l'American Society of Clinical Oncology (ASCO) en 2017. Un essai de phase II a en effet mis en évidence la faisabilité de la chirurgie chez 22 patients ayant un CBNPC opérable (NCT02259621, [21]). Quarante-trois pour cent de réponses histologiques majeures après 2 perfusions de nivolumab (anti-PD-1) étaient observés.

Pour conclure, cette étude de phase II testant une immunothérapie en néoadjuvant devrait nous permettre d'avoir des données objectives non seulement sur la faisabilité chirurgicale mais aussi des premières données d'efficacité au cours des stades localisés. 
rmr170317-R1

Remerciements

Les auteurs remercient I'Intergroupe Francophone de Cancérologie Thoracique (IFCT), promoteur académique de l'étude clinique, labellisé par l'Institut National du Cancer (InCA) et la Ligue Nationale Contre le Cancer (LNCC), ainsi que les laboratoires AstraZeneca pour leur soutien (fourniture du médicament et financement).

Les auteurs remercient également Mme Nathalie Mathiot pour son aide. 
rmr170317-R1

Références

1. DeSantis CE, Lin CC, Mariotto AB, Siegel RL, Stein KD, Kramer JL, et al. Cancer treatment and survivorship statistics, 2014. CA Cancer J Clin $2014 ; 64: 252-71$.

2. Goldstraw P, Chansky K, Crowley J, Rami-Porta R, Asamura H, Eberhardt WE, Nicholson AG, Groome P, Mitchell A, Bolejack V. The IASLC Lung Cancer Staging Project: Proposals for Revision of the TNM Stage Groupings in the Forthcoming (Eighth) Edition of the TNM Classification for Lung Cancer. J Thorac Oncol 2016; 11 : 39-51.

3. Pignon J-P, Tribodet H, Scagliotti GV, Douillard J-Y, Shepherd FA, Stephens RJ, et al. Lung adjuvant cisplatin evaluation : a pooled analysis by the LACE Collaborative Group. J Clin Oncol 2008;26:3552-9.

4. NSCLC Meta-analysis Collaborative Group. Preoperative chemotherapy for non-smallcell lung cancer : a systematic review and meta-analysis of individual participant data. Lancet $2014 ; 383: 1561-71$.

5. Pataer A, Kalhor N, Correa AM, Raso MG, Erasmus JJ, Kim ES, et al. Histopathologic response criteria predict survival of patients with resected lung cancer after neoadjuvant chemotherapy. J Thorac Oncol $2012 ; 7: 825-32$.

6. Mouillet G, Monnet E, Milleron B, Puyraveau M, Quoix E, David P, et al. Pathologic complete response to preoperative chemotherapy predicts cure in early-stage nonsmall-cell lung cancer: combined analysis of two IFCT randomized trials. J Thorac Oncol $2012 ; 7: 841-9$.

7. Hellmann MD, Chaft JE, William WN, Rusch V, Pisters KMW, Kalhor N, et al. Pathological response after neoadjuvant chemotherapy in resectable non-small-cell lung cancers: proposal for the use of major pathological response as a surrogate endpoint. Lancet Oncol $2014 ; 15: e 42-50$.

8. Iwai $\mathrm{Y}$, Ishida M, Tanaka $\mathrm{Y}$, Okazaki T, Honjo $\mathrm{T}$, Minato N. Involvement of PD-L1 on tumor cells in the escape from host immune system and tumor immunotherapy by PDL1 blockade. Proc Natl Acad Sci U S A 2002 ;99:12293-7.

9. Creelan $\mathrm{BC}$. Update on immune checkpoint inhibitors in lung cancer. Cancer Control J Moffitt Cancer Cent 2014;21:80-9.

10. Brahmer J, Reckamp KL, Baas P, Crinò L, Eberhardt WEE, Poddubskaya E, et al. Nivolumab versus Docetaxel in Advanced Squamous-Cell Non-Small-Cell Lung Cancer. N Engl J Med $2015 ; 373: 123-35$.

11. Borghaei H, Paz-Ares L, Horn L, Spigel DR, Steins M, Ready NE, et al. Nivolumab versus Docetaxel in Advanced Nonsquamous Non-Small-Cell Lung Cancer. N Engl J Med. $2015 ; 373: 1627-39$.

12. Reck M, Rodríguez-Abreu D, Robinson AG, Hui R, Csőszi T, Fülöp A, et al. Pembrolizumab versus Chemotherapy for PD-L1-Positive Non-Small-Cell Lung Cancer. N Engl J Med 2016 ;375:1823-33. 
13. Rittmeyer A, Barlesi F, Waterkamp D, Park K, Ciardiello F, von Pawel J, et al. Atezolizumab versus docetaxel in patients with previously treated non-small-cell lung cancer (OAK): a phase 3, open-label, multicentre randomised controlled trial. Lancet 2017;389:255-65.

14. Antonia SJ, Villegas A, Daniel D, Vicente D, Murakami S, Hui R, et al. Durvalumab after Chemoradiotherapy in Stage III Non-Small-Cell Lung Cancer. N Engl J Med 2017;377:1919-29

15. Weber J, Mandala M, Del Vecchio M, Gogas HJ, Arance AM, Cowey CL, et al. Adjuvant Nivolumab versus Ipilimumab in Resected Stage III or IV Melanoma. N Engl J Med 2017;377:1824-35

16. Rexer H, Retz M, Albers P. [Adjuvant study of high-risk muscle invasive urothelial carcinoma: Open-label, multicenter, randomized phase III study with atezolizumab (anti-PD-L1 antibody) as adjuvant therapy compared with observation in patients with high-risk muscle invasive urothelial carcinoma after surgical resection (IMvigor010)study AB 53/15 of the AUO]. Urol Ausg A 2016 ;55:1491-3.

17. Dogan V, Rieckmann T, Münscher A, Busch C-J. Current studies of immunotherapy in head and neck cancer. Clin Otolaryngol 2018 ;43:13-21.

18. Stewart R, Morrow M, Hammond SA, Mulgrew K, Marcus D, Poon E, et al. Identification and Characterization of MEDI4736, an Antagonistic Anti-PD-L1 Monoclonal Antibody. Cancer Immunol Res 2015 ;3:1052-62.

19. Balmanoukian AS, Antonia SJ, Hwu W-J, Hamid O, Gutierrez M, Jamal R, et al. Updated safety and clinical activity of durvalumab monotherapy in previously treated patients with stage IIIB/IV NSCLC. J Clin Oncol 2017 ;35(15_suppl):9085-9085.

20. Planchard D, Yokoi T, McCleod MJ, Fischer JR, Kim Y-C, Ballas M, et al. A Phase III Study of Durvalumab (MEDI4736) With or Without Tremelimumab for Previously Treated Patients With Advanced NSCLC: Rationale and Protocol Design of the ARCTIC Study. Clin Lung Cancer 2016;17:232-36.e1.

21. Chaft JE, Forde PM, Smith KN, Anagnostou V, Cottrell T, Taube JM, et al. Neoadjuvant nivolumab in early-stage, resectable non-small cell lung cancers. J Clin Oncol $2017 ; 35\left(15 \_s u p p l\right): 8508-08$. 
rmr170317-R1

Légendes des figures

Figure 1

Titre : Design de l'essai de phase II loNESCO évaluant l'efficacité du durvalumab en traitement néo-adjuvant des CBNPC de stade IB $(\geq 4 \mathrm{~cm})$ ou II.

Légende: $\mathrm{CBNPC}=$ Cancer bronchique non à petites cellules, $\mathrm{ECOG}=$ Eastern cooperative oncology group, PS = performance status, PD-L1 = Programmed DeathLigand 1 
Figure 1

\section{IFCT-1601 loNESCO \\ Immune Neoadjuvant Therapy in Early Stage Non-Small Cell CarcinOma}

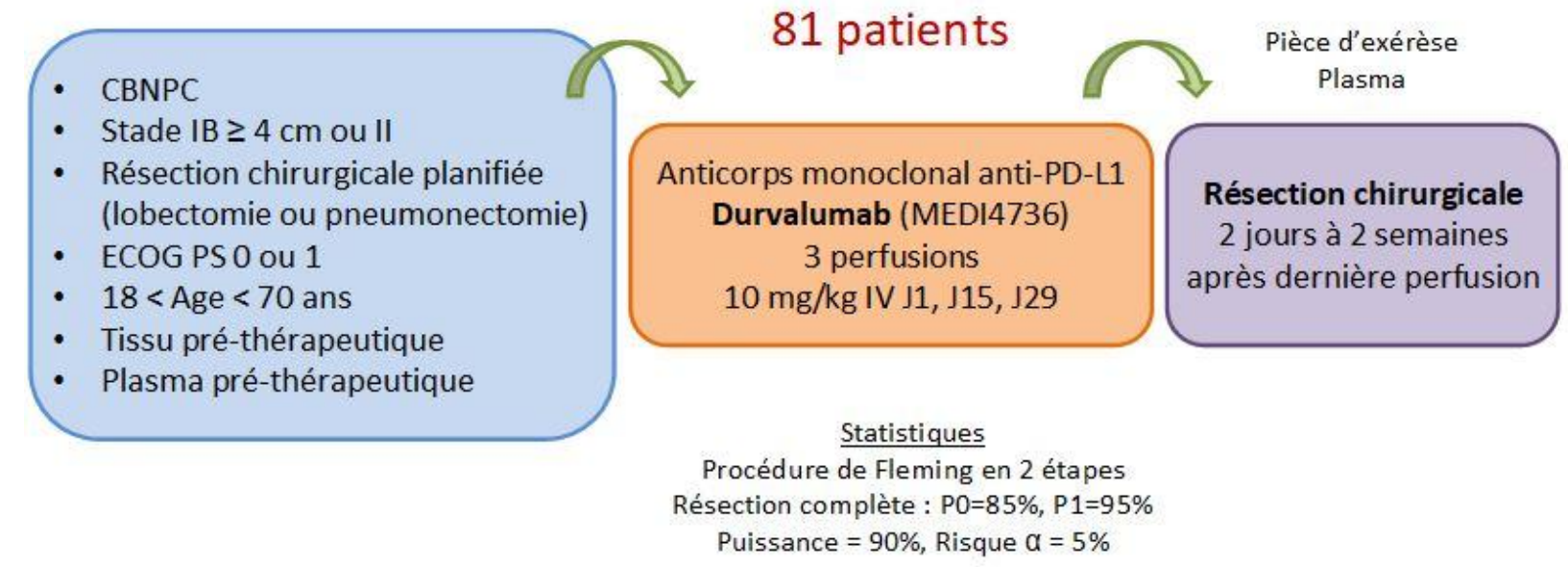

Durée d'inclusion: 12 mois (20 centres) 